\title{
Web accessibility: evaluation of a website with different semi-automatic evaluation tools
}

\author{
Edson Rufino de Souza ${ }^{\mathrm{a}, \mathrm{b}^{*}}$, and Cláudia Mont'Alvão ${ }^{\mathrm{a}^{* *}}$ \\ ${ }^{a}$ Ergonomics Laboratory, Arts and Design Department, Pontifical Catholic University of Rio de Janeiro, PUC- \\ Rio, Rua Marquês de São Vicente 225, Gavea, Rio de Janeiro, Brazil \\ ${ }^{\mathrm{b}}$ Instituto Nacional da Propriedade Industrial-INPI, Praça Mauá 7, Centro, Rio de Janeiro, Brasil
}

\begin{abstract}
It is essential that access to information must to be universal given the importance of having a truly global Internet. This study proposes to evaluate a government website with two semiautomatic accessibility evaluation tools. The results have demonstrated that the use of more than one semi-automatic assessment tool can provide enhanced results. The differences in results and presentation in the reports generated by the two tools indicate that there is more complete results by combining tests with different accessibility evaluation tools.
\end{abstract}

Keywords: web accessibility, semi-automatic evaluation tools, human-computer interaction

\section{Introduction}

It is essential that access to information must to be universal given the importance of having a truly global Internet. The advantages of universal access to Internet resources are numerous. In social terms, allows the inclusion of users in a context that allows them to work, study, relate to and entertain. In economic terms, includes these people as new customers that can be inserted in different economic niches. In psychological terms, it will generate increased selfesteem and decrease in the individual sense of powerlessness in relation to other people.

For the Internet becomes truly inclusive, it is necessary that websites are designed with a focus on accessibility. Considering usability general aspects it is possible to define that accessibility provides that everyone can use an interface with full effectiveness, efficiency and satisfaction [3]. To evaluate the accessibility of websites, there are several possible approaches. One of these approaches is to assess websites with semiautomatic tools. These tools perform a scan in the document to search for violations of web accessibility guidelines previously established. This category of tools typically is based on the Web Content Accessibility Guidelines.

This document, as proposed by the W3C standard for accessibility in web sites, is currently at version 2.0 [7], which replaced its predecessor, the WCAG 1.0 [6]. Because of the recent consolidation of WCAG 2.0 as a standard for Web accessibility, most existing tools even take into account the WCAG 1.0, even though that document known issues regarding its lack of completeness in considering the possibilities of accessibility problems [1].

\section{Method}

This study proposes to evaluate a government website with two semiautomatic accessibility evaluation tools and compare its results.

\footnotetext{
*Corresponding authors: e-mail: edson.rufino@gmail.com.
} cmontalvao@puc-rio.br. 


\subsection{About accessibility evaluation tools}

This type of tool evaluates the accessibility of a website by checking the compliance of this web site with a given set of accessibility guidelines. In most cases, these tools are based on one of the two versions of WCAG.

These tools are very useful, especially when you want to have a first impression of the level of accessibility in websites that have very complex and many pages. However, it is important to note that these tools do not have the ability to evaluate the site based on all the recommendations of the WCAG 1.0 and WCAG 2.0, since many of these recommendations require human inspection for a more effective.

For example, when WCAG 1.0 and WCAG 2.0 recommends that there must be text equivalents for all non-textual elements, the tools are able to check for alternative text for non-textual elements, but only human analysis is able to verify that there are indeed equivalence between these non-textual and textual elements.

In this study, we have considered two tools that are still based on WCAG 1.0: Hera and DaSilva. These tools have been adopted because they are two tools that are already consolidated. They present reliable and consistent results.

Furthermore, the tools that rely on WCAG 2.0 are few and mostly are still available in beta versions.

\subsection{Hera}

HERA is a tool developed by Fundación Sidar, with the aim of turning the accessibility of a website in accordance with the recommendations of the WCAG 1.0. The tool is available in twelve languages, including portuguese, spanish and english.

According to website of Fundación Sidar [2], the method used by the HERA is summed up in three phases:

1. to perform an automatic analysis of the previous page you requested on the recommendations that the tool is able to check;

2. to provide information about the errors found (detected automatically), and

3. to warn what the checkpoints of the WCAG 1.0 must be manually reviewed.

\subsection{DaSilva}

DaSilva tool also evaluates accessibility based on WCAG 1.0, and offers the option of perform an evaluation checking Brazilian guidelines for accessibility in governmental websites. In this case, the analysis based on WCAG was chosen, to maintain consistency among the tests with the two tools.

\subsection{Procedure}

Two tests were carried out using both tools Hera and DaSilva. In both ones, it was evaluated the main website's Page of INPI (Brazilian National Institute of Industrial Property). Once this is a governmental agency, its website must be accessible by virtue of law.

\section{Results}

The results of each evaluation are shown below.

\subsection{Evaluation with Hera}

After choosing a page to be evaluated and begin the process, a brief preliminary report displays a summary of the evaluation (Fig. 1).

\section{Summary}

- URL: http://www.inpi.gov.br

- Date/time: 29/08/2011 - 19:42 GMT

- Total: 165 elements

- Automatic analysis: 30 seconds

- Errors: $\mathbf{8}$ errors

- To check manually: 41 checkpoints

- Tester: (unknown)

- Navegador: Mozilla Firefox 6.0 (Windows XP)

Fig. 1: Summary of results of INPI homepage with Hera.

Doing a test on the homepage of the website of the INPI, presents the following information: the tool reports the total number of elements in the page (165) and reports the number of elements that should be reviewed manually by an expert on accessibility (41). 
Also on page summary report, a table (Fig. 2) is displayed that informs the points accordingly, errors and manually check points organized by priority levels of the WCAG 1.0 (1, 2 and 3, in order of importance according to the impact in the user experience if the recommendation is violated).

\begin{tabular}{|c|c|c|c|c|}
\hline \multicolumn{5}{|c|}{ Status of checkpoints } \\
\hline Priority & Needs checking & Pass & Fail & N/A \\
\hline $\begin{array}{c}\text { P1 } \\
\text { HERA WCAG } 1.0\end{array}$ & $9 \rho$ & $1 \checkmark$ & $1 \times$ & 6 \\
\hline $\begin{array}{c}\text { P2 } \\
\text { HERA WCAG1.0 }\end{array}$ & $19 \rho$ & $3 \checkmark$ & $5 \times$ & 2 \\
\hline $\begin{array}{c}\text { PQ } \\
\text { HERA WCAG } 1.0\end{array}$ & $13 \rho$ & $2 \checkmark$ & $2 \times$ & 2 \\
\hline
\end{tabular}

Fig. 2: Preliminary results table shown in evaluation of INPI homepage with Hera.

A detailed presentation of the results (Fig. 3) follows the structure of recommendations and checkpoints of the WCAG 1.0. It is reported the description of the recommendation, followed by tables for each of the checkpoints. In these tables, after the description of the checkpoint, is reported on page assessed for violations, in accordance elements, or whether this checkpoint must be evaluated manually. This information is categorized by element type: images, buttons, image maps areas etc..

It is informed too when the checkpoint does not apply to the page evaluated. For example, if a check- point is related to the mapping feature images, available in HTML, this point will not be assessed if there is assessed on the page.

This approach provides context and features of the evaluation results within the WCAG 1.0 at the same time informing the points that should be checked manually, which of course has to step forward for manual verification (Fig. 3).

\subsection{Evaluation with DaSilva}

In the assessment with DaSilva was also chosen the English language. However, the evaluation results are shown in Portuguese.

A table is also shown with the results organized by priority level. Nevertheless, the table shows only those elements that are errors in relation to the recommendations of WCAG and the elements that need to be checked manually (called "Avisos" - "Warnings").

Thus, there is no information about the elements that are in accordance with the recommendations, or on those who do not apply (Fig. 4).

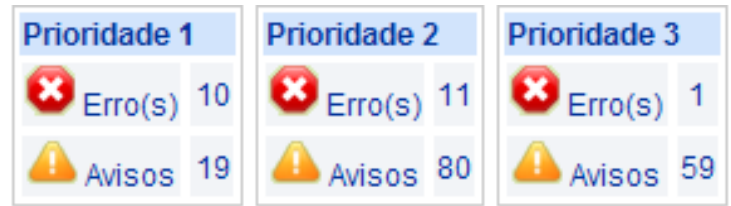

Fig. 4: Results table shown in evaluation with DaSilva.

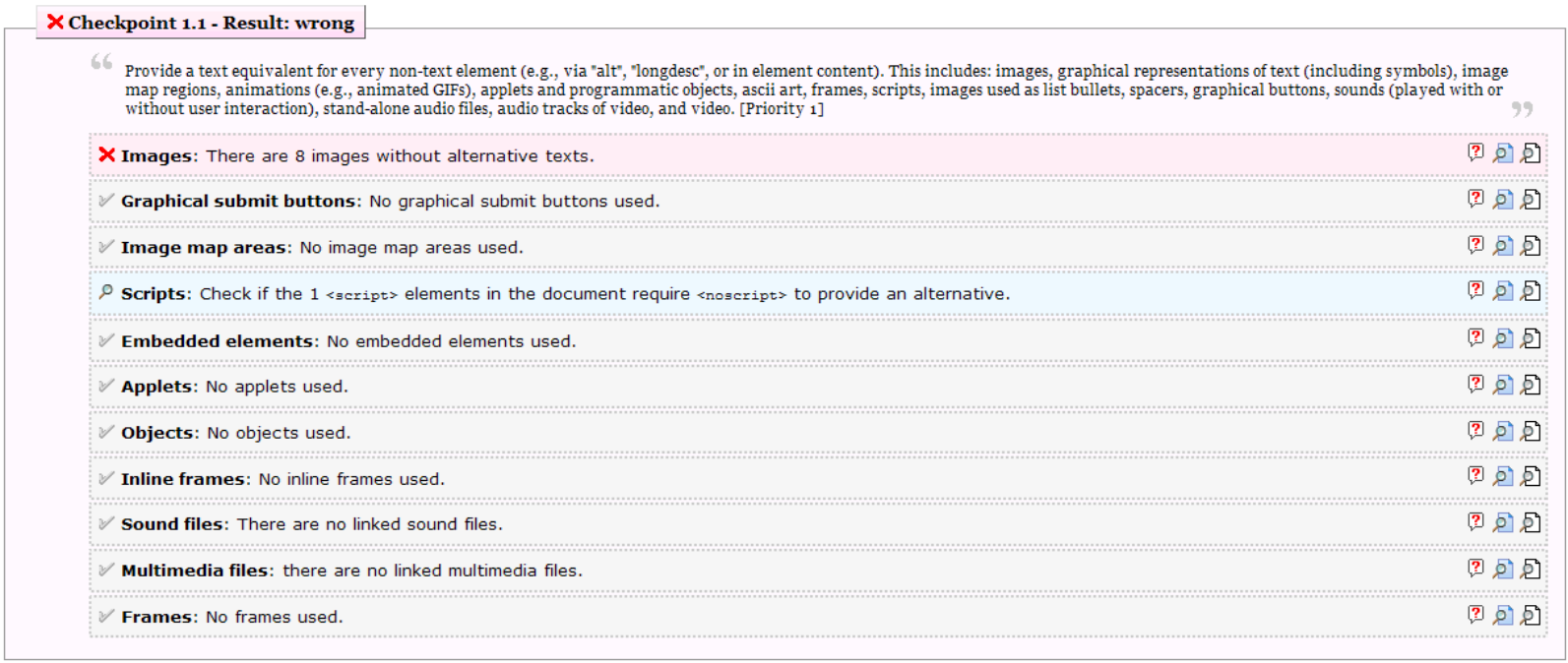

Fig. 3: Part of results of the evaluation of INPI homepage with Hera. 


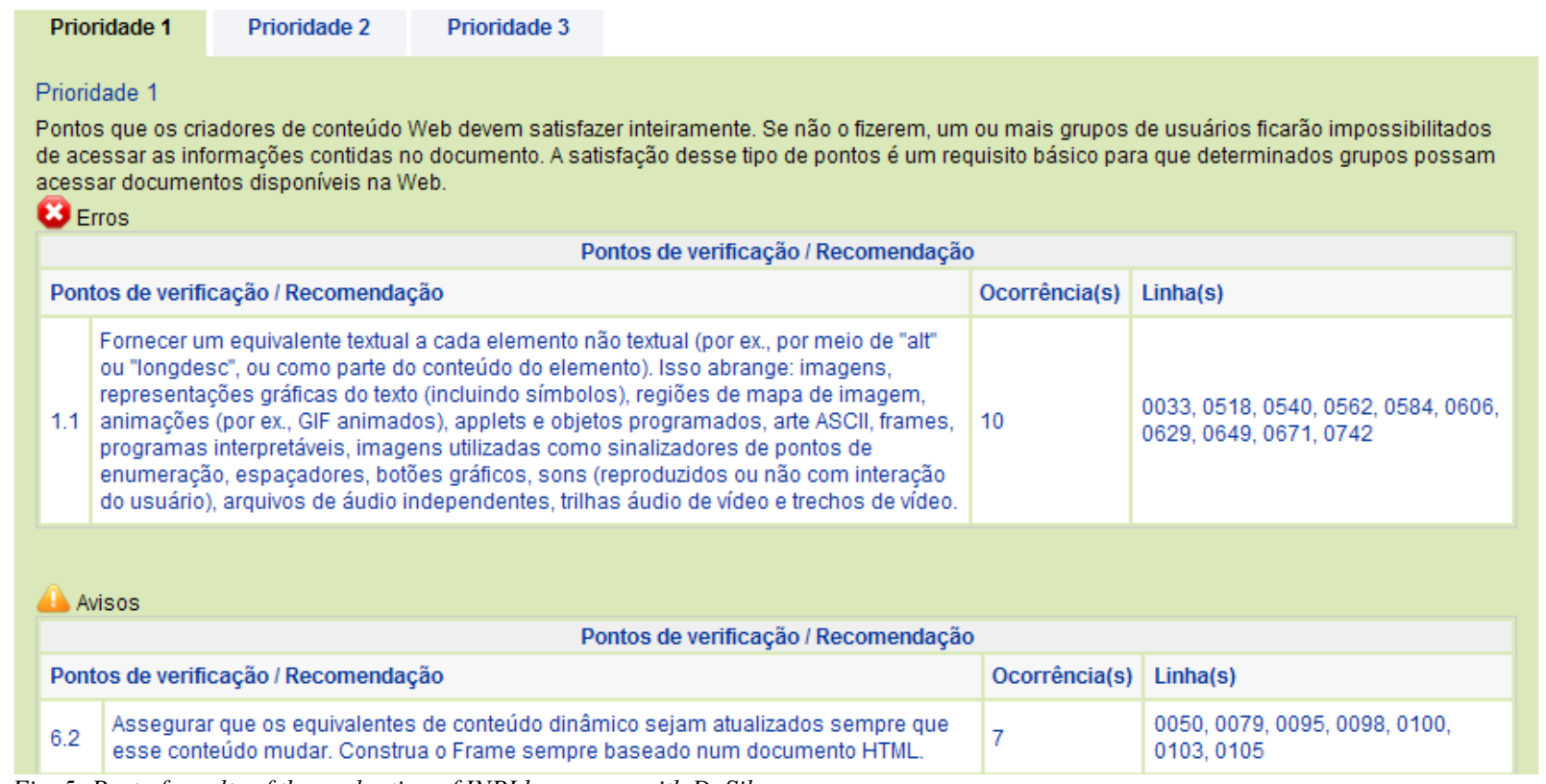

Fig. 5: Part of results of the evaluation of INPI homepage with DaSilva.

Complete results can be viewed from three tabs Priorities 1, 2 and 3 (Fig. 5).

In presenting the results, they are displayed by priority level in two tables: Errors and Warnings. In these tables, presents the checkpoints violated (or check), describing the same, the number of occurrences and the number of lines of code where they occur.

There are other types of subdivision, and by type of element.

\section{Conclusions}

The report of the two tools is significantly different. These differences are in many respects as: in the way of quantifying errors; in the form of browsing the results; and in the type of classification of elements after the evaluation.

The Hera allows a more global and didactic view of the results, including navigation elements by: only violated elements; accordingly elements; elements to be checked manually; and elements that do not apply to the page evaluated.
Nevertheless, the way of quantifying errors in $\mathrm{He}$ $r a$ is different from other tools: when he was informed in the summary of the results that had one Priority 1 error, it is not clear that there are eight occurrences of this type of error. This is cleared only if the user chooses to browse the errors.

DaSilva tool quantifies the occurrences in a more direct way: the number of errors reported in the summary of results is equal to the total number of violations. However, the lack of information on elements in accordance does not exactly about what elements have been considered accessible on the page in question.

After this analysis, it is possible to conclude that the two tools have pros and cons. Therefore, they do not have enough reliable results to be considered separately.

This study has demonstrated that the use of more than one semi-automatic assessment tool can provide enhanced and more comprehensive results. As tools of this type can only evaluate part of the items contained in WCAG 1.0, due to the need for human inspection in many of these points, best results were encountered in comparing the results of evaluations of each tool, which confirms that the sum of results offers real gains on the assessment results. 


\section{References}

[1] DRC - Disability Rights Commission (2004). The Web access and inclusion for disabled people: a formal investigation conducted by the Disability Rights Commission. London: TSO.

[2] Fundación Sidar. Revendo a Acessibilidade com Estilo (2005). On-line. Available in: http://www.sidar.org/hera/index.php.pt. Accessed in: 2011-0826.

[3] ISO - International Organization of Standardization (2003). ISO/TS 16071: Ergonomics of human-system interaction Guidance on accessibility for human-computer interfaces. Geneva, Switzerland.

[4] Nielsen, J.; Coyne, K. P. (2001). Beyond ALT Text: Making the Web Easy to Use for Users with Disabilities. California: Nielsen Norman Group.

[5] UN - United Nations. United Nations Global Audit of Web Accessibility (2006). On-line. Available in: . Accessed in: 2007-06-22.

[6] W3C - World Wide Web Consortium. Web Content Accessibility Guidelines 1.0 (1999). On-line. Available in: http://www.w3.org/TR/WCAG10/

[7] W3C - World Wide Web Consortium. Web Content Accessibility Guidelines 2.0 (2008). On-line. Available in: http://www.w3.org/TR/WCAG/ 